\title{
STVDIVM
}

Revista de Humanidades

PRENSAS DE LA UNIVERSIDAD DE ZARAGOZA FACULTAD DE CIENCIAS SOCIALES Y HUMANAS UNIVERSIDAD DE ZARAGOZA

Stvdivm 22 (2016) Zaragoza 2016 ISSN: 1137-8417 
REDACCIÓN, CORRESPONDENCIA E INTERCAMBIOS:

Studium. Revista de Humanidades

Facultad de Ciencias Sociales y Humanas

Ciudad Escolar, Carretera de Alcañiz, s/n 44003 TERUEL

Tel.: 9786181 00. Fax: 978618103

studium@unizar.es

SUSCRIPCIÓN Y PEDIDOS:

Prensas de la Universidad de Zaragoza. Edificio de Geológicas

Calle Pedro Cerbuna, 12

50009 ZARAGOZA

Tfno. 976555493 y 9763541 00. Fax: 976555493

PÁGINA WEB DE LA REVISTA:

http://studium.unizar.es

Studium. Revista de Humanidades agradece el envío de originales (artículos o reseñas), así como de libros (estudios o ediciones) para la elaboración de recensiones. La revista no mantendrá correspondencia con los autores de los artículos no aceptados para su publicación, no se verá obligada a dar explicaciones sobre las circunstancias de su rechazo ni dará a conocer los informes sobre los mismos. De no ser aceptados para su publicación, sólo serán devueltos los trabajos remitidos a petición expresa de sus autores, para lo cual deberán remitir previamente el franqueo necesario.

(C) De los autores

(C) De la presente edición, Prensas de la Universidad de Zaragoza

Edita: Prensas de la Universidad de Zaragoza y Facultad de Ciencias Sociales y Humanas, Universidad de Zaragoza, con la ayuda económica del Vicerrectorado de Política Científica de la Universidad de Zaragoza. Periodicidad anual.

PRECIO DE CADA NÚMERO: 12 Euros

Ilustración de la cubierta: Mirambel, celosías (Foto: Peña Verón)

Coordinación, diagramación y corrección de estilo: María Luz Rodrigo Estevan

ISSN: $1137-8417$

Depósito Legal: Z-2751-90

Impresión: Servicio de Publicaciones de la Universidad de Zaragoza 


\title{
DIRECCIÓN
}

María Luz Rodrigo Estevan (UZ)

\author{
SECRETARÍA \\ Pedro Luis Hernando Sebastián (UZ) \\ CONSEJO DE REDACCIÓN \\ Pedro Luis Hernando Sebastián (UZ) \\ María Luz Rodrigo Estevan (UZ) \\ José Manuel Latorre Ciria (UZ) \\ Ana M. Rivera (UNED, Madrid) \\ Frédéric Duhart (MU, Donostia) \\ Juan A. Tarancón (UZ) \\ Xavier Medina (UOC, Barcelona)
}

\section{CONSEJO CIENTÍFICO}

Ricardo J. Ávila Palafox (Estudios del Hombre, U. Guadalajara, Jalisco, México)

Carlos Barros Guimerans (Historia Medieval, U. Santiago de Compostela)

Elvira Burgos Díaz (Filosofía, U. Zaragoza)

Marcela Cubillos Poblete (Historia, U. La Serena, Chile)

Francisco Javier Díez de Revenga (Literatura Española, U. Murcia)

Elbia H. Difabio (Griego, U.N. Cuyo, Argentina)

Javier Esparcia Pérez (Geografía, U. Valencia)

Claudio García Turza (Lengua Española, U. La Rioja)

Xavier Gil Pujol (Historia Moderna, U. Barcelona)

Alfredo Jimeno Martínez (Prehistoria, U. Complutense)

Isabel González Turmo (Antropología Social, U. Sevilla)

Emma Liaño Martínez (Historia del Arte, U. Rovira i Virgili)

M. ${ }^{a}$ Mercedes López Suárez (Artes, U.N. Cuyo, Argentina)

Javier Martín Arista (Filología Inglesa, U. La Rioja)

Javier Pons Díez (Piscología Social, U. Valencia)

Inés Praga Terente (Filología Inglesa, U. Burgos)

Alberto Sabio Alcutén (H. Contemporánea, U. Zaragoza)

Norma Vasallo (C. de la Mujer, U. La Habana, Cuba)

Alicia Yllera Fernández (Filología Francesa, UNED) 



\section{STVDIVM 22 (2016)}

Stvdivm. Revista de Humanidades

Prensas de la Universidad de Zaragoza

Universidad de Zaragoza. ISSN: 1137-8417

\section{ÍNDICE}

\section{Estudios}

La controversia del Ara de Victoria

Ignacio Jesús ÁlvAREZ SORIA

Historia espiritual y sensibilidad estética:

El Otoño de la Edad Media, de Johan Huizinga

Ricardo PÉrez Gómez.....

Aprender a hablar y escribir en castellano en el Virreinato del Perú: la construcción de identidades transculturales en los dibujos didácticos de Guamán Poma de Ayala (c.1616)

Delfín OrTEga SÁNCHEZ.

La introducción del liberalismo inglés en Barcelona:

Lo bello y lo sublime, de Manuel Casamada Ignasi Roviró Alemany

Del fracaso a la Aurora: los orígenes sombrios del saber

Lorena ROJAS PARMA

Une table metabistorique en Calabre.

Vivants, morts, saints et anges en partageant la nourriture

Alfonsina BeLLIO

Compartir alimentos en el Michoacán del siglo XV

Rodolfo FERnÁndeZ y Daría DERAGA.

Fiesta y comida ritual a los santos que enferman en Coatetelco, Morelos (México) 
La olla, la fuente y el plato.

Distintas maneras de compartir en Argentina

Patricia AgUIRRE

Société et jeunes couples à Tlemcen et son hawz:

entre prescriptions et proscriptions alimentaires

Mustapha GUENAOU

El ñandú en Uruguay: el consumo alimentario

de un ave nativa percibida como exótica

Gustavo LABORDE...

\section{Notas y reseñas}

Mediterranean Algarve. Tradition, Product and Cuisine, de Maria Manuel Valagão, Vasco Célio y Bertílio Gomes Antoni GonzÁlez Novell y F. Xavier Medina

Sharing Food, de Eric de Garine, María Luz Rodrigo,

Christine Raimond y F. Xavier Medina, coords.

Luis CANTARERO ABAD

Las terapias-placebo. Posibilidades, limitaciones, perspectivas, de Eduardo Aznar Martínez

Fernando SERRANO LARRÁYOZ

SUMARIOS

NORMAS PARA LA PUBLICACIÓN DE ORIGINALES

BOLETINES DE SUSCRIPCIÓN E INTERCAMBIO 


\section{STVDIVM 22 (2016)}

Stvdivm. Revista de Humanidades

Prensas de la Universidad de Zaragoza

Universidad de Zaragoza. ISSN: 1137-8417

\section{TABLE OF CONTENTS}

\section{Articles}

The Controversy of Victoria's Altar

Ignacio Jesús ÁlvAREZ SORIA

Spiritual History and Aesthetic Sensibility: Johan Huizinga's

The Autumn of the Middle Ages

Ricardo PÉREZ GÓMEZ.

Learning to Speak and Write in Castilian Spanish in the

Viceroyalty of Peru: The Construction of Transcultural

Identities in the Didactic Drawings of Guamán Poma de Ayala

(c. 1616)

Delfín ORTEGa SÁNCHEZ

The Introduction of English Liberalism in Barcelona:

Manuel Casamada's The Beautiful and the Sublime

Ignasi Roviró AlEMANY

From Failure to Aurora: The Dark Origins of Knowledge

Lorena ROJAS PARMA

A Metabistorical Table in Calabria. The Living, the Dead, Saints and Angels Sharing Food

Alfonsina BELLIO

Sharing Food in Michoacan during the 15 ${ }^{\text {th }}$ Century

Rodolfo FERnÁndeZ y Daría DERAGa ....

Celebrations and Ritual Foods for the Saints that Cause

Illnesses in Coatetelco, Morelos (México)

Mayán CERVANTES 
The Pot, the Platter and the Dish:

Various Ways of Sharing in Argentina

Patricia AgUIRRE

Society and Young Couples in Tlemcen and Its Peri-Urban

Area: Between Alimentary Prescriptions and Proscriptions

Mustapha GueNAOU

The Nandú in Uruguay:

The Consumption of an Indigenous Species Perceived as Exotic

Gustavo LABORDE.

\section{Notes \& Reviews}

Mediterranean Algarve. Tradition, Product and Cuisine, Maria Manuel Valagão, Vasco Célio y Bertílio Gomes

Antoni González Novell and F. Xavier Medina

Sharing Food, Eric de Garine, María Luz Rodrigo,

Christine Raimond and F. Xavier Medina, eds.

Luis CANTARERO ABAD

Las terapias-placebo. Posibilidades, limitaciones, perspectivas, Eduardo Aznar Martínez

Fernando SERRANO LARRÁYOZ.

Abstracts

GUIDELINES FOR CONTRIBUTORS 